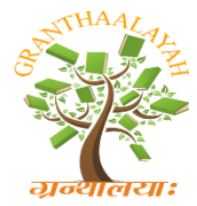

\author{
INTERNATIONAL JOURNAL OF RESEARCH - \\ GRANTHAALAYAH \\ A knowledge Repository
}

Science

\title{
LOCAL SEARCH OPTIMIZATION: A COMPARISON OF ALGORITHMS FOR NONHYPERBOLIC TRAVEL-TIME ANALYSIS
}

\author{
Nelson Ricardo Coelho Flores Zuniga *1 \\ ${ }^{*}$ Departamento de Geofísica, Instituto de Astronomia, Geofísica e Ciências Atmosféricas, \\ Universidade de São Paulo, Brazil
}

\begin{abstract}
In the last decade, many works compared nonhyperbolic multiparametric travel-time approximations to perform velocity analysis. In these works, some analyses were accomplished, such as accuracy analysis and objective function analysis. However, no previous works compared the optimization algorithms to perform the inversion procedure concerning the processing time and the accuracy of each algorithm. As the shifted hyperbola showed the best results among the unimodal approximations in previous works, it was selected to be used in a comparison with five local search optimization algorithms. Each algorithm was compared concerning the accuracy by the minimization of the calculated curve to the observed curve. The travel-time curves tested here are conventional (PP) and converted wave (PS) reflection events from an offshore model. With this set of tests, it is possible to define which optimization algorithm presents the most reliable result when used with the shifted hyperbola equation concerning the processing time and the accuracy.
\end{abstract}

Keywords: Optimization Algorithms; Nonhyperbolic; Travel-Time; Seismic Inversion; Multicomponent.

Cite This Article: Nelson Ricardo Coelho Flores Zuniga. (2019). "LOCAL SEARCH OPTIMIZATION: A COMPARISON OF ALGORITHMS FOR NONHYPERBOLIC TRAVELTIME ANALYSIS." International Journal of Research - Granthaalayah, 7(4), 321-328. https://doi.org/10.29121/granthaalayah.v7.i4.2019.914.

\section{Introduction}

Several nonhyperbolic travel-time approximations were developed in last decades essentially for the velocity analysis step $[4,7,24,25,28,38]$, with different characteristics and proposals. These approximations have been tested and compared along the last decade for different situations and conditions, with different kinds of analysis [3, 17, 40-44]. Comparison of accuracy of the traveltime approximations, processing time of each approach and objective function analysis are some examples of tests performed so far. However, there is no works published in previous years that compare the optimization algorithm for this kind of problem. 
The approximation proposed by Malovichko [25] is the first nonhyperbolic multiparametric approach proposed after the hyperbola equation [15]. In the previous works, it showed very good results and a unimodal behavior, what makes it able to be used with a local search optimization algorithm with no need of a multi start procedure. Thus, it is possible to perform the inversion minimizing the calculated curve with this equation to the observed nonhyperbolic travel-time curve.

The tests were performed with five local search algorithm to perform the optimization with the shifted hyperbola approximation [25], and therefore it were analyzed which one showed the best results concerning the accuracy and the processing time.

\section{Materials and Methods}

Dix (1955) proposed initially the hyperbola equation (Equation 1), where $x$ is the vector of offsets, $t_{0}$ is the zero-offset travel-time and $v$ is the RMS (Root Mean Square) velocity.

$$
t=\sqrt{t_{0}^{2}+\frac{x^{2}}{v^{2}}}
$$

However, it was not valid for layered media with large offsets, what leads Malovichko [25] to propose an approximation known as shifted hyperbola (Equation 2) which was also studied and derived by Castle [8-9] and de Bazelaire [14]. This approximation was developed to control the effect of large offsets in inhomogeneous media using the $S$ parameter.

$$
t=t_{0}^{2}\left(1-\frac{1}{S}\right)+\frac{1}{S} \sqrt{t_{0}^{2}+\frac{S x^{2}}{v^{2}}}
$$

The $S$ parameter (Equation 3) depends on the $\mu_{4}$ and $\mu_{2}$ by the relation $S=\mu_{4} / \mu_{2}^{2}$, where $\mu_{j}(j=$ $2,4)$ is the $j$ th velocity momentum (Equation 3 ).

$$
\mu_{j=} \sum_{k=1}^{n} t_{k} v_{k}^{j} / \sum_{k=1}^{n} t_{k}
$$

and $v_{k}$ is the interval velocity of the $k$ th layer and $t_{k}$ is the travel-time of the $k$ th layer.

Once the optimization aims to minimize a function to find its least error, it is necessary to use a method which solves each kind of problem more efficiently [18]. Among many optimization algorithms [32], five methods were selected to be used aiming to reach the minimum value of the objective function in this work.

IMFIL (Implicit Filtering) is an implementation in Matlab of the implicit filtering algorithm, a local search algorithm with a deterministic sampling method for bound-constrained optimization. This kind of algorithm is useful for to minimize functions which are non-smooth, noisy, random or discontinuous [16, 21, 39]. 
The NEWUOA is the Powell's model-based algorithm implemented in Fortran for derivative-free optimization. In this algorithm the inputs and lower bounds for the trust-region radius, and the number of function values to be used for interpolating the quadratic model [30-31].

NOMAD is an implementation in C++ of the LTMADS [5] and ORTHOMADS [2] method based in the concept of three approaches to handle general constraints: extreme barrier, filter and progressive barrier [1,6]. This algorithm was proposed to solve nonlinear, nonsmooth, noisy optimization problems, and it works by a combination of the number of points evaluated minimum mesh size and the acceptable number of consecutives trials that failed to make an improvement in the value. A related implementation of this algorithm is a Matlab set of functions capable to solve bound-constrained, linear or nonlinear optimization problems that presents continuous, discrete, and categorical variables.

FMINSEARCH (Find Minimum Search) is an implementation in Matlab of the Nelder-Mead algorithm [29] which is based on the simplex method [22, 33]. This local search algorithm is focused in the use of a simplex, a polytope of $n+1$ vertices in $n$ dimensions with edges of the same size, and it is useful for unconstrained optimization problems [10, 23$]$.

SID-PSM (Simplex Derivative - Pattern Search Method) is a local search algorithm implemented in Matlab based on a pattern search method with the pool step guided by simplex derivatives [11, 12]. This kind of algorithm was created to solve unconstrained and constrained problems, and each search step is based on the optimization of quadratic surrogate models [13]. As the optimization methods shown previously are local search algorithms, it is necessary to use the multi-start procedure which is based on perform several inversions starting from different sets of initial points [19-20, 34-35].

To analyze the residual travel-time error is necessary to compare the difference between the observed curves to the calculated curve. In this work it is used the nonhyperbolic travel-time approximation known as shifted hyperbola proposed by Malovichko [25]. Previous works compared travel-time approximations among them for VTI (Vertical Transverse Isotropy) media [3, 17, 42], strong heterogeneity [41], and for OBN (Ocean Bottom Nodes) data [40, 43-44]. However, this work aims to compare different optimization algorithms to improve the results obtained with shifted hyperbola equation in previous works. Each inversion routine, for each optimization algorithm was composed by 1000 inversions. It were performed 100 inversion routines, to understand the stability of the problem. Then, it was computed the mean accuracy and the mean processing time to perform the analyses, once the problem showed to be significantly stable.

The proposed model to be used in this work (Table 1) is an offshore layered geological structure with a sandstone reservoir $\left(V_{P}=2952 \mathrm{~m} / \mathrm{s}\right.$ and $\left.V_{S}=1593 \mathrm{~m} / \mathrm{s}\right)$ sealed by a shale layer ( $5^{\text {th }}$ layer). It was modeled considering the ray tracing for PP and PS events [26-27, 37]. The variation of physical properties as P-wave velocity, S-wave velocity and the ratio between Vp and Vs are shown in the Figure 1, where it can be clearly observed their relation with the depth. In Figure 2, it is possible to observe the simulated ray tracing which generated the travel-time curves and the seismograms used in this work to study the PP and the PS reflection event. 
Table 1: The parameters of the Model: Layer thickness $(\Delta \mathrm{z}), \mathrm{P}$-wave velocity $\left(V_{P}\right), \mathrm{S}$-wave

\begin{tabular}{|l|l|l|l|l|}
\hline \multicolumn{6}{|c|}{ velocity $\left(V_{S}\right)$ and $V_{P} / V_{S}$ ratio. } \\
\hline Water & 1052 & 1500 & 0 & - \\
\hline 1 & 298 & 1930 & 643 & 3.00 \\
\hline 2 & 559 & 2127 & 1134 & 1.88 \\
\hline 3 & 311 & 2357 & 1202 & 1.96 \\
\hline 4 & 161 & 2554 & 1390 & 1.84 \\
\hline 5 & 77 & 2733 & 1403 & 1.95 \\
\hline
\end{tabular}
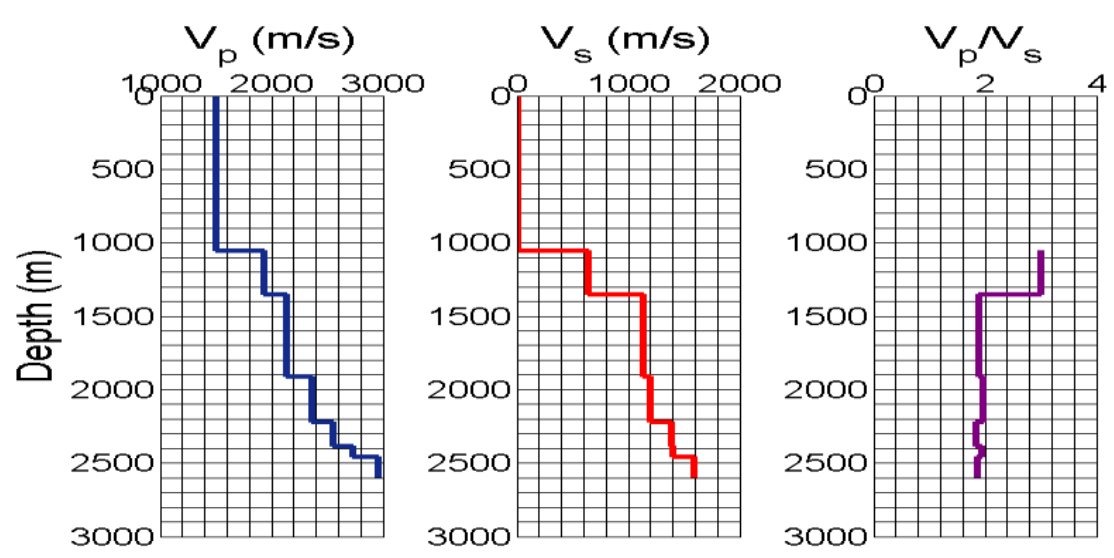

Figure 1: P-wave velocity $\left(V_{P}\right), \mathrm{S}$-wave velocity $\left(V_{S}\right)$ and $V_{P} / V_{S}$ ratio profiles of the Model.
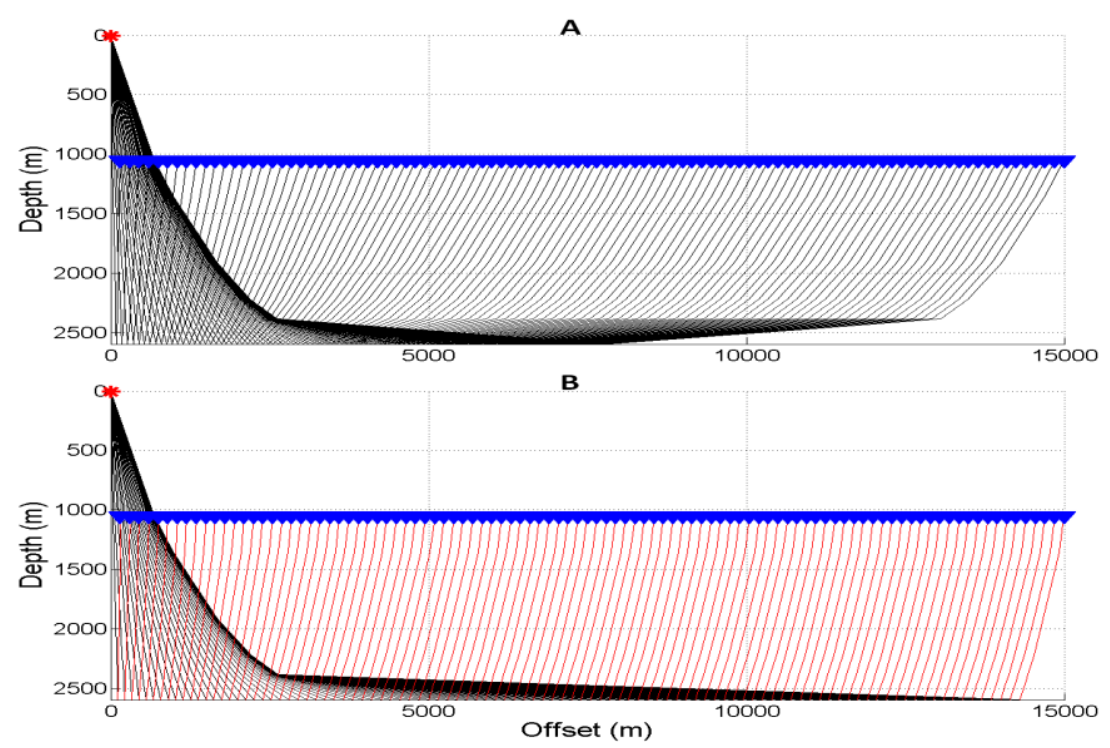

Figure 2: Ray tracing of the (A) PP wave reflection event and (B) PS wave reflection event of the 2Model.

\section{Results and Discussions}

The IMFIL algorithm presented the worst result for the PP (Figure 3A) and for the PS (Figure 3B) wave reflection event. NEWUOA algorithm showed the second highest errors for PP and PS 
events, even it being significantly lower than the errors showed by IMFIL algorithm. Almost indistinctly better, it can be observed the NOMAD algorithm with the third best results for the conventional and the converted reflection events. Just a little better than the previous algorithm, FMINSEARCH algorithm showed the second best results for both reflection events. SID-PSM is the algorithm which showed the best results for the PP and PS reflection events.

Concerning the processing time (Table 2), the IMFIL showed the lowest one for the both reflection events. Less than 9\% slower to perform the inversion procedure, NEWUOA and NOMAD algorithms showed, respectively, the second and the third lower processing times. With a processing time around 12\% higher than the IMFIL and a little slower than NEWUOA and NOMAD, FMINSEARCH showed the second best processing times. The highest processing time among all algorithms tested here was shown by SID-PSM algorithm, almost twice the processing time of the FMINSEARCH algorithm. In general, the PS reflection event showed higher processing times than the conventional reflection event, as it was predicted due to the higher complexity of this kind of event.

Table 2: The mean processing time (in seconds) to perform an inversion routine with each optimization algorithm for both reflection events.

\begin{tabular}{|l|l|l|}
\hline Algorithms & PP event & PS event \\
\hline IMFL & 74.2 & 77.4 \\
\hline NEWUOA & 78.8 & 80.3 \\
\hline NOMAD & 80.4 & 82.0 \\
\hline FMINSEARCH & 83.1 & 86.7 \\
\hline SID-PSM & 150.6 & 157.1 \\
\hline
\end{tabular}
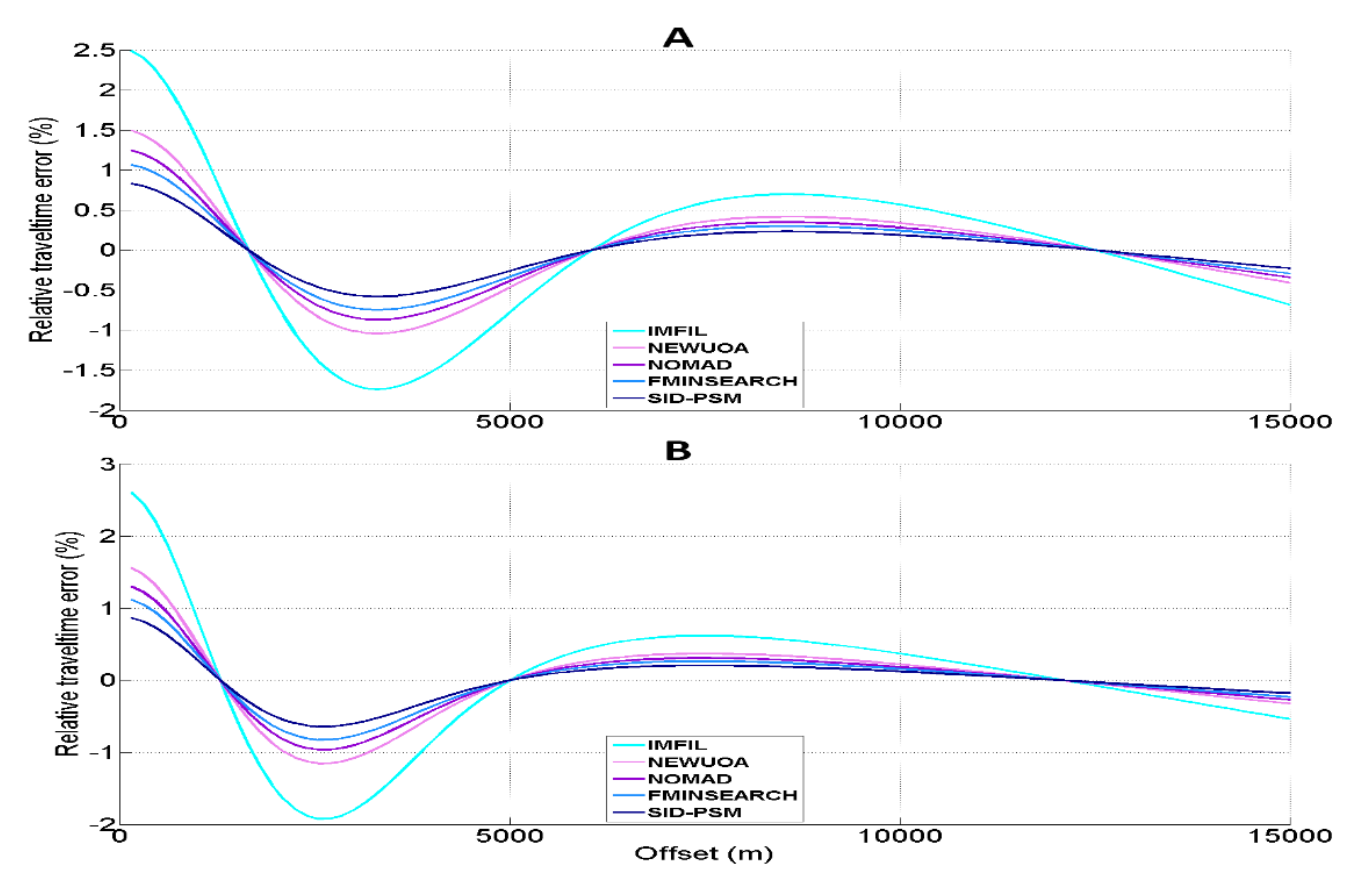

Figure 3: Relative errors in travel-time between the observed curve and the calculated curve with the approximation proposed by Malovichko [25] for each optimization algorithm for the (A) PP wave reflection event and (B) PS wave reflection event. 


\section{Conclusions and Recommendations}

The IMFIL algorithm showed the worst set of results concerning the residual error and showed the lowest processing time, what makes this algorithm the worst for this kind of problem even with a good processing time. NEWUOA algorithm showed the second worst set of results for both reflection events, with the second higher residual error and the second best processing time. A very similar result was shown by the NOMAD algorithm, with a strongly similar result to the NEWUOA algorithm. However, for details, NOMAD showed the third best result with the third best processing time.

For both reflection events, FMINSEARCH algorithm showed the second best results very close to SID-PSM algorithm. Even FMINSEARCH algorithm showing the second worst processing time it is just little higher than the previous algorithms tested here and almost twice faster than SIDPSM algorithm, what leads the FMINSEARCH to be the best optimization algorithm to be used with this nonhyperbolic multiparametric travel-time approximation.

\section{Acknowledgements}

This study was funded by Coordenação de Aperfeiçoamento de Pessoal de Nível Superior - Brasil (CAPES) (grant number 001) and by Conselho Nacional de Desenvolvimento Científico e Tecnológico - Brasil (CNPq) (grant number 001).

\section{References}

[1] Abramson MA. Pattern search algorithm for mixed variable general constrained optimization problems. PhD Thesis, Rice University, 2002, 180.

[2] Abramson MA, Audet C, Dennis JE, LeDigabel S. (2009) A deterministic MADS instance with orthogonal directions. SIAM Journal on Optimization, 20, 2009, 948-966.

[3] Aleixo R, Schleicher J. Traveltime approximations for q-P waves in vertical transversely isotropic media. Geophysical Prospectting, 58, 2010,191-201.

[4] Alkhalifah T, Tsvankin I. Velocity analysis for transversely isotropic Media. Geophysics, 60, 1995,1550-1566.

[5] Audet C, Dennis JE. Mesh adaptive direct search algorithms for constrained optimization. SIAM Journal on Optimization, 17, 2006,188-217.

[6] Audet C, Dennis JE. A progressive barrier for derivative-free nonlinear programming. SIAM Journal on Optimization, 20, 2009, 445-472.

[7] Blias E. Long-offset NMO approximations for a layered VTI model: Model study. 79th Annual International Meeting, Society of Exploration Geophysics, Expanded Abstract, Houston, 2009, 3745-3749.

[8] Castle RJ. A theory of normal moveout. Geophysics, 59, 1994, 983-999.

[9] Castle RJ. Shifted hyperbolas and normal moveout. 58th Annual International Meeting, Society of Exploration Geophysics, Expanded Abstract, 1988, 894-896.

[10] Coxeter HSM. Regular Polytopes. Methuen, 1948, 321.

[11] Custódio AL, Vicente LN. Using sample and simplex derivatives in pattern search methods. SIAN Journal on Optimization, 2007, 537-555.

[12] Custódio AL, Vicente LN. SID-PSM: A pattern search method guided by simplex derivatives for use in derivative-free optimization. Technical report of the Department of Mathematics of the University of Coimbra, 2008. 
[13] Custódio AL, Rocha H, Vicente LN. Incorporating minimum Frobenius norm models in direct search. Computational Optimizing and Applications, 46, 2010, 265-278.

[14] de Bazelaire E. Normal moveout revisited: Inhomogeneous media and curved interfaces. Geophysics, 53, 1988, 143-157.

[15] Dix CH. Seismic velocities from surface measurements. Geophysics, 20, 1955, 68-86.

[16] Gilmore P, Kelley CT. An implicit filtering algorithm for optimization of functions with many local minima. SIAM Journal on Optimization, 5, 1995, 269-285.

[17] Golikov P, Stovas A. Accuracy comparison of nonhyperbolic moveout approximations for qPwaves in VTI media. Journal of Geophysics and Engineering, 9, 2012, 428-432.

[18] Horst R, Pardalos PM, Thoai NV. Introduction to global optimization. 2nd ed. Dordrecht: Kluwer Academic Publusher, 2000, 354.

[19] Kan HAHG, Timmer GT. Stochastic optimization method part I: Clustering methods. Mathematical Programing, 39, 1987a, 27-56.

[20] Kan HAHG, Timmer GT. Stochastic optimization method part II: Multi-level methods. Mathematical Programing, 39, 1987b, 57-78.

[21] Kelley CT. Implicit Filtering, $1^{\text {st }}$ ed., Raleigh, 2011, 158.

[22] Lagarias JC, Reeds JA, Wright PE. Convergence properties of the Nelder-Mead simplex method in low dimensions. SIAM Journal on Optimization, 9, 1998,112-147.

[23] Lewis RM, Torczon V, Trosset MW. Direct search methods: Then and now. ICASE Report, 2000.

[24] Li XY, Yuan J. Converted wave imaging in inhomogeneous, anisotropic media: Part I. Parameter estimation. 63rd EAGE Conference, Amsterdam, The Netherlands., Expanded Abstract, 1, 2001, 109.

[25] Malovichko AA. A new representation of the traveltime curve of reflected waves in horizontally layered media. Applied Geophysics (in Russian), 91, 1978, 47-53.

[26] Margrave GF. New seismic modelling facilities in Matlab. CREWES Research Report, 12, 2000.

[27] Margrave GF. Numerical methods explorations seismology with algorithms in Matlab. CREWES Research Report, 2003.

[28] Muir F, Dellinger J. A practical anisotropic system, in SEP-44. Stanford Exploration Project, 1985, 55-58.

[29] Nelder JA, Mead RA. A simplex method for function minimization. The Computer Journal, 7 , 1965, 308-313.

[30] Powell MJD. The NEWUOA software for unconstrained optimization without derivatives. LargeScale Nonlinear Optimization, 2006, 255-297.

[31] Powell MJD. Development of NEWUOA for minimization without derivatives. IMA Journal of Numerical Analysis, 28, 2008, 649-664.

[32] Rios LM, Sahinidis NV. Derivative-free optimization: A review of algorithms and comparison of softwares implementations. Journal of Global Optimization, 56, 2013, 1247-1293.

[33] Spendley W, Hext GR, Himsworth FR. Sequential Application of Simplex Designs in Optimization and Evolutionary Operation. Technometrics, 4, 1962, 441-461.

[34] Telraky T, Sotirov R. Multi-start approach to global conic optimization. ISE Archives of Working Papers, 2010.

[35] Telraky T, Sotirov R. Multi-start approach for an integer determinant maximization problem. Optimization, 61, 2013, 101-114.

[36] Thomsen L. Weak elastic anisotropy. Geophysics, 51, 1986, 1954-1966.

[37] Thorbecke JW, Draganov D. Finite-difference modeling experiment for seismic interferometry. Geophysics, 76, 2012, H1-H18.

[38] Ursin B, Stovas A. Traveltime approximations for a layered transversely isotropic medium. Geophysics, 71, 2006, 23-33.

[39] Wislow TA, Trew RJ, Gilmore P, Kelley, CT. Simulated performance optimization of gas mesfet amplifiers. Conference on Advances Concepts in High Speed Semiconductor Device Circuits, Expanded Abstract, 1991. 
[40] Zuniga NRCF. Análise comparativa de aproximações não-hiperbólicas dos tempos de trânsito de dados sísmicos multicomponente utilizando tecnologia OBN. Master's Thesis, Universidade de São Paulo, Brazil, 2017, 86.

[41] Zuniga NRCF, Bokhonok O, Diogo LA. Comparison of nonhyperbolic travel-time approximations for multicomponent seismic data. In: 14th SBGf Congress, Expanded Abstract, Rio de Janeiro, Brazil, 2015, 1176-1181.

[42] Zuniga NRCF, Molina EC, Prado RL. Inversion of multicomponent seismic data for VTI medium using the globalized Nelder-Mead optimization algorithm. In: 3th EAGE/SBGf Workshop, Expanded Abstract. Rio de Janeiro, Brazil, 2016a.

[43] Zuniga NRCF, Molina EC, Prado RL. Inversion of multicomponent seismic data of the Santos Basin. Far East Hydrocarbons, Expanded Abstract, 2016b.

[44] Zuniga NRCF, Molina EC, Prado RL. Comparison of travel-time approximations for unconventional reservoirs from Santos Basin, Brazil. Revista Brasileira de Geofísica, 35, 2017, 271-286.

\footnotetext{
*Corresponding author.

E-mail address: nelson.zuniga@iag.usp.br
} 